\title{
Das US-amerikanische Discovery-Verfahren im Rahmen deutscher gerichtlicher Auseinandersetzungen
}

Eine Untersuchung unter rechtsvergleichenden Gesichtspunkten sowie unter besonderer Berücksichtigung des Verfahrens nach 28 U.S.C. § 1782 (a) als Beweisbeschaffungsmöglichkeit für Patentstreitigkeiten vor deutschen Gerichten

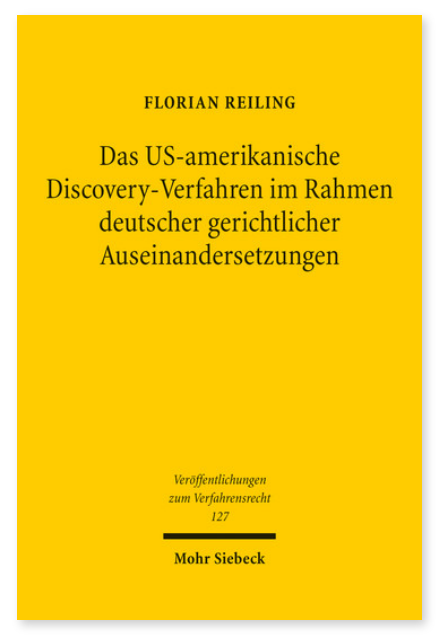

2016. XXII, 424 Seiten. VVerfR 127

ISBN 978-3-16-154686-0

DOI 10.1628/978-3-16-154686-0

eBook PDF 89,00€

ISBN 978-3-16-154552-8

fadengeheftete Broschur 89,00€
Die Beweisermittlung oder Beschaffung von Beweisen, die in der Sphäre des jeweiligen Verfahrensgegners liegen, gestaltet sich für den Anspruchsteller häufig schwierig. Dies gilt umso mehr, wenn sich die maßgeblichen Beweise beispielsweise in den USA befinden und das entsprechende Gerichtsverfahren in Deutschland rechtshängig ist.

Florian Reiling stellt das US-amerikanische Beweisverfahren nach 28 USC 1782 (a) dar, das umfassende Möglichkeiten bietet, um der relevanten Beweisstücke habhaft zu werden. Die Ausgestaltung des Verfahrens nach 28 USC 1782 (a) als Variante der US-amerikanischen Beweisermittlung führt allerdings auch dazu, dass sich insbesondere auf zivilprozessualer Ebene gewisse Zweifel und Risiken ergeben, ob eine derart weitreichende Beweisermittlung durch das deutsche Zivilprozessrecht gedeckt ist. Diese Zweifel wirken sich letztlich auch auf der Ebene der Einführung und der Ebene der Verwertbarkeit der Beweise aus und können im Einzelfall dazu führen, dass auf bestimmte in den USA erlangte Beweisstücke nicht zurückgegriffen werden kann.

Florian Reiling untersucht das Verfahren nach 28 USC 1782 (a) nicht nur hinsichtlich dessen einzelner Voraussetzungen, sondern nimmt sich vor allem auch der geschilderten zivilprozessualen Bedenken an und versucht diese aufzulösen. Ein besonderer Schwerpunkt liegt dabei auf der Betrachtung von patentrechtlichen Auseinandersetzungen vor deutschen Gerichten und den damit verbundenen Besonderheiten im Hinblick auf einen Rückgriff auf das Verfahren nach 28 USC 1782 (a).

Florian Reiling Geboren 1984; Studium der Rechtswissenschaften mit wirtschaftswissenschaftlicher Zusatzausbildung an der Universität Bayreuth; 2010 erstes, 2012 zweites Juristisches Staatsexamen; Referendariat in Mönchengladbach mit Stationen in Hanoi und Paris; seit 2012 Associate im Bereich IP/Litigation bei einer internationalen Anwaltssozietät in Düsseldorf.

Jetzt bestellen:

https://mohrsiebeck.com/buch/das-us-amerikanische-discovery-verfahren-im-rahmen-deutscher-gerichtlicher-

auseinandersetzungen-9783161546860?no_cache $=1$

order@mohrsiebeck.com

Telefon: +49 (0)7071-923-17

Telefax: $+49(0) 7071-51104$ 